Bull. Austral. Math. Soc.

VoL. 75 (2007) [469-480]

\title{
REMARKS ON THE SEMIVARIATION OF VECTOR MEASURES WITH RESPECT TO BANACH SPACES.
}

\author{
OScar Blasco
}

Suppose that $L^{q}(\nu) \widehat{\otimes}_{\gamma_{q}} Y=L^{q}(\nu, Y)$ and $X \widehat{\bigotimes}_{\Delta_{p}} L^{p}(\mu)=L^{p}(\mu, X)$. It is shown that any $L^{p}(\mu)$-valued measure has finite $L^{2}(\nu)$-semivariation with respect to the tensor norm $L^{2}(\nu) \widehat{\otimes}_{\Delta_{p}} L^{p}(\mu)$ for $1 \leqslant p<\infty$ and finite $L^{q}(\nu)$-semivariation with respect to the tensor norm $L^{q}(\nu) \widehat{\otimes}_{\gamma_{q}} L^{p}(\mu)$ whenever either $q=2$ and $1 \leqslant p \leqslant 2$ or $q>\max \{p, 2\}$. However there exist measures with infinite $L^{q}$-semivariation with respect to the tensor norm $L^{q}(\nu) \widehat{\otimes}_{\gamma_{q}} L^{p}(\mu)$ for any $1 \leqslant q<2$. It is also shown that the measure $m(A)=\chi_{A}$ has infinite $L^{q}$-semivariation with respect to the tensor norm $L^{q}(\nu) \widehat{\bigotimes}_{\gamma_{q}} L^{p}(\mu)$ if $q<p$.

\section{INTRODUCTION}

Let $Z$ be a Banach space and let $m: \Sigma \rightarrow Z$ be a vector measure defined on a $\sigma$-algebra $\Sigma$ of subsets of $\Omega$. We write $|m|$ for the variation of the measure

$$
|m|(A)=\sup \left\{\sum_{j=1}^{k}\left\|m\left(A_{j} \cap A\right)\right\|: A_{j} \text { pairwise disjoints }, k \in \mathbb{N}\right\}
$$

and denote, for $1 \leqslant p<\infty$, the $p$-variation of the measure

$$
\|m\|_{p}=\sup \left\{\left(\sum_{j=1}^{k}\left\|m\left(A_{j}\right)\right\|^{p}\right)^{1 / p}: A_{j} \text { pairwise disjoints }, k \in \mathbb{N}\right\} .
$$

We also write $\|m\|=\sup _{A \in \Sigma}\|m(A)\|$, which is equivalent to the semivariation of the vector measure $m$, that is

$$
\|m\| \approx \sup \left\{\left|\left\langle z^{*}, m\right\rangle\right|(\Omega):\left\|z^{*}\right\|=1\right\} .
$$

Suppose that $X, Y$ is a Banach spaces and let $\tau$ be a norm on $X \otimes Y$ such that $\|x \otimes y\|_{\tau} \leqslant C\|x\|\|y\|$ for $x \in X, y \in Y$ and denote $X \widehat{\otimes}_{\tau} Y$ the completion under such a

Received 28th November, 2006

The author was partially supported by Proyecto MTM 2005-08350.

Copyright Clearance Centre, Inc. Serial-fee code: 0004-9727/07 \$A2.00+0.00. 
norm. Given a vector measure $m: \Sigma \rightarrow Y$ defined on a $\sigma$-algebra $\Sigma$ of subsets of $\Omega$, R. Bartle (see $[2,7]$ ) introduced the notion of $X$-semivariation of $m$ in $X \otimes_{\tau} Y$ given by

$$
\beta_{X}(m, \tau, Y)(A)=\sup \left\{\left\|\sum_{j=1}^{k} x_{j} \otimes m\left(A \cap A_{j}\right)\right\|_{\tau}\right\}
$$

for every $A \in \Sigma$ where the supremum is taken over $\left\|x_{j}\right\| \leqslant 1, A_{j}$ pairwise disjoints sets in $\Sigma$ and $k \in \mathbb{N}$. We shall denote

$$
\beta_{X}(m, \tau, Y)=\sup _{A \in \Sigma} \beta_{X}(m, \tau, Y)(A) .
$$

It is clear that

$$
\|m\| \leqslant \beta_{X}(m, \tau, Y) \leqslant\|m\|_{1} .
$$

If $X \widehat{\bigotimes}_{\varepsilon} Y$ and $X \widehat{\bigotimes}_{\pi} Y$ stand for the injective and projective tensor norms respectively, then one always has

$$
\|m\| \leqslant \beta_{X}(m, \varepsilon, Y) \leqslant \beta_{X}(m, \tau, Y) \leqslant \beta_{X}(m, \pi, Y) \leqslant\|m\|_{1} .
$$

It is well-known and easy to see that actually $\beta_{X}(m, \varepsilon, Y)=\|m\|$.

In [7] Jefferies and Okada developed a theory of integration of $X$-valued functions with respect to $Y$-valued measures of bounded $X$-semivariation in the case of completely separated tensor norms.

We shall be concerned with some interesting examples of norms coming from the theory of vector-valued functions: Throughout the paper $\left(\Omega_{1}, \Sigma_{1}, \mu\right)$ and $\left(\Omega_{2}, \Sigma_{2}, \nu\right)$ are finite measure spaces, $1 \leqslant p, q<\infty$ and the Banach spaces will be either $Y=L^{p}(\mu)$ or $X=L^{q}(\nu)$. We define $\gamma_{q}$ and $\Delta_{p}$ the norms on $L^{q}(\nu) \otimes Y$ and $X \otimes L^{p}(\mu)$ identified as subspace of $L^{q}(\nu, Y)$ and $L^{p}(\mu, X)$, that is to say

$$
L^{q}(\nu) \widehat{\bigotimes}_{\gamma_{q}} Y=L^{q}(\nu, Y), \quad X \widehat{\bigotimes_{\Delta_{p}}} L^{p}(\mu)=L^{p}(\mu, X)
$$

In the case $p=q$ the $L^{p}(\nu)$-semivariation of $L^{p}(\mu)$-valued measures with respect to the topology $\tau_{p}$ such that $L^{p}(\mu) \widehat{\bigotimes}_{\tau_{p}} L^{p}(\nu)$ becomes $L^{p}(\mu \times \nu)$ for the product measure was studied in $[8,9]$.

In particular, if both $X=L^{q}(\nu)$ and $Y=L^{p}(\mu)$ then $L^{q}(\nu) \widehat{\otimes}_{\Delta_{p}} L^{p}(\mu)$ and $L^{q}(\nu) \widehat{\bigotimes}_{\gamma_{q}} L^{p}(\mu)$ coincide with the spaces of measurable functions $f: \Omega_{1} \times \Omega_{2} \rightarrow \mathbb{R}$ such that

$$
\left(\int_{\Omega_{1}}\left(\int_{\Omega_{2}}|f(x, y)|^{q} d \nu(y)\right)^{p / q} d \mu(x)\right)^{1 / p}<\infty
$$

and

$$
\left(\int_{\Omega_{2}}\left(\int_{\Omega_{1}}|f(x, y)|^{p} d \mu(x)\right)^{q / p} d \nu(x)\right)^{1 / q}<\infty
$$


In this paper we shall try to understand better the difference between the classical semivariation or variation of a $L^{p}(\mu)$-valued measure $m$ and the $L^{q}(\nu)$-semivariation with respect to the norms $\Delta_{p}, \gamma_{q}$ and $\pi$.

Let us establish the main results of the paper. Our first result establishes the following descriptions of the $L^{q}$-semivariation of $L^{p}$-valued measures with respect to the projective tensor norm, where we denote $L^{p}=L^{p}([0,1])$ for $1 \leqslant p \leqslant \infty$.

THEOREM 1.1. Let $1 \leqslant p, q \leqslant \infty$ and let $m: \Sigma \rightarrow L^{p}([0,1])$ be a vector measure. Then
(i) $\beta_{L^{p^{\prime}}}\left(m, \pi, L^{p}\right) \approx\|m\|_{1}$
$1 \leqslant p \leqslant \infty$.
(ii) $\beta_{L^{2}}\left(m, \pi, L^{p}\right) \approx\|m\|_{1}, \quad 1<p<\infty$.
(iii) $\beta_{L^{2}}\left(m, \pi, L^{1}\right) \approx\|m\|$.

This result shows that $L^{2}$-valued measures are of finite $L^{2}$-semivariation on $L^{2} \bigotimes_{\pi} L^{2}$ if and only if they are of finite variation.

It was noticed in $[8]$ that any $L^{2}$-valued measure is of bounded $L^{2}$-semivariation with respect to $L^{2}([0,1]) \widehat{\bigotimes}_{\tau_{2}} L^{2}([0,1])$, in other words $\beta_{L^{2}}\left(m, \Delta_{2}, L^{2}\right) \approx\|m\|$.

On the other hand $\beta_{L^{q}}\left(m, \pi, L^{1}\right)=\beta_{L^{q}}\left(m, \Delta_{1}, L^{1}\right)$. Hence Theorem 1.1 shows that $\beta_{L^{2}}\left(m, \Delta_{1}, L^{1}\right)=\|m\|$.

Let us just point out that this implies

$$
\beta_{L^{2}}\left(m, \Delta_{p}, L^{p}\right) \approx\|m\|, 1 \leqslant p \leqslant 2
$$

due to the simple observation

$$
\beta_{L q(\nu)}\left(m, \Delta_{p_{1}}, L^{p_{1}}(\mu)\right) \leqslant C \beta_{L^{q}(\nu)}\left(m, \Delta_{p_{2}}, L^{p_{2}}(\mu)\right) \quad p_{1} \leqslant p_{2} .
$$

We shall present another alternative proof that cover all the cases and gives an alternative proof of the known case $p=q=2$ and extend (1) as follows.

THEOREM 1.2. Let $1 \leqslant p<\infty$ and let $m: \Sigma \rightarrow L^{p}([0,1])$ be a vector measure. Then

$$
\beta_{L^{2}}\left(m, \Delta_{p}, L^{p}\right) \approx\|m\| .
$$

The question which now arises is whether or not there exist $L^{p}$-valued measures with $\beta_{L^{q}(\nu)}\left(m, \Delta_{p}, L^{p}(\mu)\right)=\infty$ if $q \neq 2$. In [7] examples of $L^{p}([0,1])$-valued measures of infinite $L^{p}([0,1])$-semivariation in $L^{p}([0,1]) \widehat{\bigotimes}_{\tau_{p}} L^{p}([0,1])$ were obtained for the values $p \neq 2$. For $1 \leqslant p<2$ the approach was much simpler than for $p>2$ and the example in this case relies on the existence of a non absolutely summing operator from $\ell^{1} \rightarrow \ell^{p}$ for $p>2$ (see $[8,9])$.

We shall use the relationship between the tensor norms $\gamma_{q}$ and $\Delta_{p}$ to get other examples. Recall that Minkowski's inequality gives $L^{p}\left(\mu, L^{q}(\nu)\right) \subseteq L^{q}\left(\nu, L^{p}(\mu)\right)$ for $p \leqslant q$ 
and $L^{q}\left(\nu, L^{p}(\mu)\right) \subseteq L^{p}\left(\mu, L^{q}(\nu)\right)$ for $q \leqslant p$. Hence

$$
\begin{aligned}
& \beta_{L^{q}(\nu)}\left(m, \gamma_{q}, L^{p}(\mu)\right) \leqslant \beta_{L^{q}(\nu)}\left(m, \Delta_{p}, L^{p}(\mu)\right), \quad p \leqslant q, \\
& \beta_{L^{q}(\nu)}\left(m, \Delta_{p}, L^{p}(\mu)\right) \leqslant \beta_{L^{q}(\nu)}\left(m, \gamma_{q}, L^{p}(\mu)\right), \quad q \leqslant p .
\end{aligned}
$$

Also using general techniques, similar to those used in [8] one can show that for $1 \leqslant p \leqslant \infty$ and $1 \leqslant q<2$ there exist $L^{p}(\mu)$-valued measures $m$ such that $\beta_{L^{q}(\nu)}\left(m, \gamma_{q}, L^{p}(\mu)\right)=\infty$. This, in particular, using the estimate (3), shows the existence of measures for which $\beta_{L^{q}(\nu)}\left(m, \Delta_{p}, L^{p}(\mu)\right)=\infty$ if $1 \leqslant q<2, p \leqslant q$, completing and extending the case $p=q$.

THEOREM 1.3 . Let $1 \leqslant p \leqslant \infty$ and let $m: \Sigma \rightarrow L^{p}([0,1])$ be a vector measure. Then

(i) $\beta_{L^{2}}\left(m, \gamma_{2}, L^{p}\right) \approx\|m\|, \quad 1 \leqslant p \leqslant 2$.

(ii) $\beta_{L^{q}}\left(m, \gamma_{q}, L^{p}\right) \approx\|m\|, \quad \max \{p, 2\}<q$.

This gives that any measure has $\beta_{L^{q}}\left(m, \gamma_{q}, L^{p}\right)<\infty$ for $q>p \geqslant 2$. However in the last section it is shown that the $L^{p}([0,1])$-valued measure $m_{p}(A)=\chi_{A}$ has infinite $L^{q}([0,1])$-semivariation in $L^{q}([0,1]) \widehat{\otimes}_{\gamma_{q}} L^{p}([0,1])$ for $q<p$.

\section{Bounded $X$-SEMIVARIATION}

We start by the following characterisation of the bounded $X$-semivariation.

Taking into account that $X \widehat{\otimes}_{\pi} Y \subset X \widehat{\otimes}_{\tau} Y$, then $\left(X \widehat{\otimes}_{\tau} Y\right)^{*}$ can be regarded as a subspace of the space of bounded operators $\mathcal{L}\left(Y, X^{*}\right)$. Moreover $\|u\| \leqslant\|u\|_{\left(X \widehat{\otimes}_{r} Y\right)^{*}}$ for any $u \in\left(X \widehat{\boldsymbol{\otimes}}_{\tau} Y\right)^{*}$, where the duality is given by

$$
\left\langle u, \sum_{j=1}^{k} x_{j} \otimes y_{j}\right\rangle=\sum_{j=1}^{k}\left\langle u\left(y_{j}\right), x_{j}\right\rangle .
$$

ThEOREM 2.1. Let $m: \Sigma \rightarrow Y$ be a vector measure. Then

$$
\beta_{X}(m, \tau, Y) \approx \sup \left\{\|u \circ m\|_{1}: u \in \mathcal{L}\left(Y, X^{*}\right),\|u\|_{\left(X \widehat{\otimes}_{r} Y\right)^{*}} \leqslant 1\right\} .
$$

Proof: Let $\left(x_{j}\right)$ be a bounded sequence in $X$ and $\left(A_{j}\right)$ be a sequence of pairwise disjoint sets in $\Sigma$. Consider, for $k \in \mathbb{N}$, the $X$-valued simple function $\phi=\sum_{j=1}^{k} x_{j} \chi_{A_{j}}$ and
denote

$$
\phi \bigotimes_{\tau} m(A)=\sum_{j=1}^{k} x_{j} \otimes m\left(A \cap A_{j}\right) \in X \otimes Y
$$

Clearly this defines a new $X \widehat{\bigotimes}_{\tau} Y$-valued measure and one can rewrite

$$
\beta_{X}(m, \tau, Y)=\sup \left\{\left\|\phi \bigotimes_{\tau} m\right\|: \phi \in \mathcal{S}(X),\|\phi\|_{\infty} \leqslant 1\right\}
$$


We now write the semivariation of $\phi \bigotimes_{\tau} m$ using duality, that is to say

$$
\begin{aligned}
\left\|\phi \bigotimes_{\tau} m\right\| & \approx \sup \left\{|\langle u, \phi \otimes m\rangle|(\Omega):\|u\|_{\left(X{\overline{\Theta_{\tau}}}_{Y}\right)^{*}} \leqslant 1\right\} \\
& =\sup \left\{\sum_{j=1}^{k}\left|\left\langle u \circ m\left(A_{j}\right), x_{j}\right\rangle\right|:\left(A_{j}\right) \text { pairwise disjoint, }\|u\|_{\left(X \widehat{\otimes}_{r} Y\right)^{*}} \leqslant 1\right\},
\end{aligned}
$$

which, taking supremum over $\left\|x_{j}\right\| \leqslant 1$, gives

$$
\begin{aligned}
\beta_{X}(m, \tau, Y) & \approx \sup \left\{\sum_{j=1}^{k}\left\|u \circ m\left(A_{j}\right)\right\|:\left(A_{j}\right) \text { pairwise disjoint, }\|u\|_{\left(X \widehat{\otimes}_{r} Y\right)^{*}} \leqslant 1\right\} \\
& \approx \sup \left\{\|u \circ m\|_{1}: u \in \mathcal{L}\left(Y, X^{*}\right),\|u\|_{\left(X \widehat{\otimes}_{\tau} Y\right)^{*}} \leqslant 1\right\} .
\end{aligned}
$$

Let us see the formulation of Theorem 2.1 in the case $\tau=\Delta_{p}$ or $\tau=\gamma_{q}$.

It is well known that for $1<p, q<\infty$ and $1 / p^{\prime}+1 / p=1,1 / q+1 / q^{\prime}=1$ and for $X, Y$ such that $X^{*}$ and $Y^{*}$ have the Radon-Nikodym property (see [6]) then

$$
\left(L^{q}(\nu) \widehat{\bigotimes}_{\gamma_{q}} Y\right)^{*}=L^{q^{\prime}}(\nu) \widehat{\otimes}_{\gamma_{q^{\prime}}} Y^{*}
$$

and

$$
\left(X \widehat{\bigotimes_{\Delta_{p}}} L^{p}(\mu)\right)^{*}=X^{*} \widehat{\bigotimes_{\Delta_{p^{\prime}}}} L^{p^{\prime}}(\mu) .
$$

Now for each $f \in L^{p^{\prime}}\left(\mu, X^{*}\right)$ we can define the operators $u_{f}: L^{p}(\mu) \rightarrow X^{*}$ and $v_{f}: X \rightarrow L^{p^{\prime}}(\mu)$ given by

$$
\left\langle u_{f}(\phi), x\right\rangle=\int_{\Omega}\langle f(t), x\rangle \phi(t) d \mu(t)
$$

and

$$
v_{f}(x)=\langle f, x\rangle \text {. }
$$

Of course $\left(v_{f}\right)^{*}=u_{f}$ and $\left(u_{f}\right)^{*}=v_{f}$ if $X$ is reflexive.

THEOREM 2.2. Let $1<p, q<\infty, X=L^{q}(\nu)$ and $Y=L^{p}(\mu)$. If $m: \Sigma \rightarrow L^{p}(\mu)$ is a vector measure then

$$
\begin{gathered}
\beta_{L^{q}(\nu)}\left(m, \Delta_{p}, L^{p}(\mu)\right)=\sup \left\{\left\|u_{f} \circ m\right\|_{1}:\|f\|_{L^{p^{\prime}}\left(\mu, L^{q^{\prime}}(\nu)\right)} \leqslant 1\right\} \\
\beta_{L^{q}(\nu)}\left(m, \gamma_{q}, L^{p}(\mu)\right)=\sup \left\{\left\|v_{g} \circ m\right\|_{1}:\|g\|_{L^{q^{\prime}}\left(\nu, L^{p^{\prime}}(\mu)\right)} \leqslant 1\right\}
\end{gathered}
$$

Proof: In the case $Y=L^{p}(\mu)$ and $X=L^{q}(\nu)$ for $1<q, p<\infty$ the elements $u: L^{p}(\mu) \rightarrow L^{q^{\prime}}(\nu)$ such that $u \in\left(L^{q}(\nu) \widehat{\otimes}_{\Delta_{p}} L^{p}(\mu)\right)^{*}$ can be seen as $u=u_{f}$ for some $f \in L^{p^{\prime}}\left(\mu, L^{q^{\prime}}(\nu)\right)$, that is $u: L^{p}(\mu) \rightarrow L^{q}(\nu)$ is given by

$$
u(\phi)(y)=\int_{\Omega_{1}} f(x, y) \phi(x) d \mu(x) .
$$


Then (6) follows from Theorem 2.1 in this case.

Similarly the elements $u: L^{p}(\mu) \rightarrow L^{q^{\prime}}(\nu)$ such that $u \in\left(L^{q}(\nu) \widehat{\bigotimes}_{\gamma_{q}} L^{p}(\mu)\right)^{*}$ can be seen as $u=v_{g}$ for some $g \in L^{q^{\prime}}\left(\nu, L^{p^{\prime}}(\mu)\right)$ and now

$$
u(\psi)(y)=\langle g, \psi\rangle=\int_{\Omega_{1}} g(y, x) \psi(x) d \mu(x) .
$$

Again (6) follows from Theorem 2.1.

\section{Proof of the MAIN THEOREMS}

We use first the characterisation in Theorem 2.1 to get the following corollaries.

Corollary 3.1. Let $m: \Sigma \rightarrow Y$ be a vector measure and $X$ a Banach space. Then

$$
\beta_{X}(m, \pi, Y) \approx \sup \left\{\|u \circ m\|_{1}: u \in \mathcal{L}\left(Y, X^{*}\right),\|u\| \leqslant 1\right\} .
$$

We use the notation $\Pi_{p}(X, Y)$ for the space of $p$-summing operators from $X$ into $Y$ and write $\pi_{p}(u)$ for the $p$-summing norm. The reader is referred to [5] for the basics in the theory of summing operators.

Corollary 3.2. Let $Y$ be a Grothendieck space, that is, $\Pi_{1}(Y, H)=\mathcal{L}(Y, H)$ for any Hilbert space $H$. Then

$$
\beta_{H}(m, \pi, Y) \approx\|m\| .
$$

ProOF: Note that $\sum m\left(A_{j}\right)$ is an unconditionally convergent series in $Y$ for any sequence of pairwise disjoint sets $A_{j}$. Now for any operator from $u: Y \rightarrow H$ one has $\sum\left\|u\left(m\left(A_{j}\right)\right)\right\| \leqslant K_{G}\|u\|\|m\|$, where $K_{G}$ is the Grothendieck constant. Now use Corollary 3.1 .

Proof of Theorem 1.1: (i) Let $Y=L^{p}$ and $X=L^{p^{\prime}}$ then choosing $u=I d$ : $L^{p} \rightarrow\left(L^{p^{\prime}}\right)^{*}$, one concludes that $\|u \circ m\|_{1}=\|m\|_{1}$. This shows $\beta_{L^{p}}\left(m, \pi, L^{p}\right)=\|m\|_{1}$

(ii) follows from the following observation: If $X^{*}$ is isomorphic to a complemented subspace of $Y$ then $\beta_{X}(m, \pi, Y) \approx\|m\|_{1}$.

Indeed, assume $i d: Y \rightarrow Y$ factors through $X^{*}$ as $i d=u_{1} \circ u_{2}$ where $u_{2}: Y \rightarrow X^{*}$ and $u_{1}: X^{*} \rightarrow Y$ are bounded operators. Now observe that $\|m\|_{1} \leqslant\left\|u_{1}\right\|\left\|u_{2} \circ m\right\|_{1}$ and use Corollary 3.1.

Now use that the space Rad is complemented in $L^{p}([0,1])$ and isomorphic to $\ell^{2}$ (see [5, Theorem 1.12]) and therefore to $L^{2}$, to conclude that

$$
\beta_{L^{2}}\left(m, \pi, L^{p}([0,1])\right) \approx\|m\|_{1}, 1<p<\infty .
$$

(iii) follows from Corollary 3.2.

We now recall a lemma that we shall need in the sequel. 
LEMMA 3.3. (i) Suppose that $1<q<\infty$ and let $Y$ be a Banach space such that $Y^{*} \in R N P$. If $u: Y \rightarrow L^{q^{\prime}}(\nu)$ belongs to $\left(L^{q}(\nu) \widehat{\otimes}_{\gamma_{q}} Y\right)^{*}$ then $\pi_{q^{\prime}}(u) \leqslant\|u\|_{\left(L^{q}(\nu) \widehat{\otimes}_{\gamma_{q}} Y\right)^{*}}$.

(ii) Let $1<p<\infty$ and let $X$ be a Banach space such that $X^{*} \in R N P$. If $u: L^{p}(\mu) \rightarrow X^{*}$ belongs to $\left(X \widehat{\otimes}_{\Delta_{p}} L^{p}(\mu)\right)^{*}$ then $\pi_{p^{\prime}}\left(u^{*}\right) \leqslant\left\|u^{*}\right\|_{\left(X \widehat{\otimes}_{\Delta_{p}} L^{p}(\mu)\right)^{*}}$.

Proof: (i) It is well known (see [5, Example 2.11]) that if $g \in L^{q^{\prime}}\left(\nu, Y^{*}\right)$ then $v_{g}: Y \rightarrow L^{q^{\prime}}(\nu)$ given by $v_{g}(y)=\langle g, y\rangle$ is $q^{\prime}$-summing and $\pi_{q^{\prime}}\left(v_{g}\right) \leqslant\|g\|_{L^{\prime}(\nu, Y)}$. Now use that, under the assumptions, $\left(L^{q}(\nu) \widehat{\bigotimes}_{\gamma_{q}} Y\right)^{*}=L^{q^{\prime}}\left(\nu, Y^{*}\right)$ and $u=v_{g}$ for certain $g \in L^{q^{\prime}}\left(\nu, Y^{*}\right)$.

(ii) Note that $u=u_{f}$ for some $f \in L^{p^{\prime}}\left(\mu, X^{*}\right)$. Hence $v_{f}=u^{*}: X^{* *} \rightarrow L^{p}(\mu)$ is $p^{\prime}$-summing and $\pi_{p^{\prime}}\left(u^{*}\right) \leqslant\|f\|_{L^{p^{\prime}}\left(\mu, X^{*}\right)}=\|u\|_{\left(L^{q}(\nu) \bar{\otimes}_{\gamma q} Y\right)^{*}}$.

Proof of Theorem 1.2: The case $p=1$ is included in (iii) Theorem 1.1.

Assume now $1<p<\infty$ and let $m: \Sigma \rightarrow L^{p}$ be a vector measure. Given $u: L^{p}$ $\rightarrow L^{2}$ with $u \in\left(L^{2} \widehat{\otimes}_{\Delta_{p}} L^{p}\right)^{*}$ we can use (ii) in Lemma 3.3 to conclude that there exist $f \in L^{p^{\prime}}\left([0,1], L^{2}\right)$ such that $v_{f}: L^{2} \rightarrow L^{p^{\prime}}$ given by $\phi \rightarrow \int_{0}^{1} \phi(y) f(x, y) d y$ is $p^{\prime}$-summing and $u=u_{f}=\left(v_{f}\right)^{*}$. Hence, using [5, Theorem 2.21], one has that $\left(v_{f}\right)^{*}=u: L^{p} \rightarrow L^{2}$ is 1-summing. Therefore

$$
\left\|u_{f} \circ m\right\|_{1} \leqslant C\left\|u_{f}\right\|\|m\| \leqslant C\|f\|_{L^{\prime}\left([0,1], L^{2}\right)}\|m\| .
$$

Let us mention another useful lemma.

Lemma 3.4. ([1, Proposition 6]) Suppose that $Y$ is a Banach space of finite cotype $r$ and let $\sum_{j} y_{j}$ be an unconditionally convergent series in $Y$.

(i) If $r=2$ then there exist $\left(\alpha_{j}\right) \in \ell^{2}$ and a sequence in $\left(y_{j}^{\prime}\right) \subset Y$ such that $y_{j}=\alpha_{j} y_{j}^{\prime}$ and

$$
\begin{array}{r}
\sum_{j}\left|\alpha_{j}\right|^{2} \leqslant \sup _{\left\|y^{*}\right\|=1} \sum_{j}\left|\left\langle y_{j}, y^{*}\right\rangle\right|, \\
\sup _{\left\|y^{*}\right\|=1} \sum_{j}\left|\left\langle y_{j}^{\prime}, y^{*}\right\rangle\right|^{2}
\end{array}
$$

(ii) If $r>2$ then for any $q>r$ there exist $\left(\alpha_{j}\right) \in \ell^{q}$ and a sequence in $\left(y_{j}^{\prime}\right) \subset Y$ such that $y_{j}=\alpha_{j} y_{j}^{\prime}$ and

$$
\begin{aligned}
\left(\sum_{j}\left|\alpha_{j}\right|^{q}\right)^{1 / q} & \leqslant\left(\sup _{\left\|y^{*}\right\|=1} \sum_{j}\left|\left\langle y_{j}, y^{*}\right\rangle\right|\right)^{1 / q} . \\
\left(\sup _{\left\|y^{*}\right\|=1} \sum_{j}\left|\left\langle y_{j}^{\prime}, y^{*}\right\rangle\right|^{q^{\prime}}\right)^{1 / q^{\prime}} & \leqslant\left(\sup _{\left\|y^{*}\right\|=1} \sum_{j}\left|\left\langle y_{j}, y^{*}\right\rangle\right|\right)^{1 / q^{\prime}} .
\end{aligned}
$$

Proof: (i) Let $T: c_{0} \rightarrow Y$ such that $T\left(e_{j}\right)=y_{j}$. Note that $\mathcal{L}\left(c_{0}, Y\right)=\Pi_{2}\left(c_{0}, Y\right)$ for any cotype 2 space $Y$. Now apply [5, Lemma 2.23] to the sequence $\left(e_{j}\right)$ which satisfies 
$\sup \left\{\sum_{j}\left|\left\langle e_{j}, z\right\rangle\right|:\|z\|_{\ell^{1}}=1\right\}$ to conclude that $T\left(e_{j}\right)=y_{j}=\alpha_{j} y_{j}^{\prime}$ with the desired properties.

(ii) Repeat the proof using now $L\left(c_{0}, Y\right)=\Pi_{q}\left(c_{0}, Y\right)$ for any $q>r$ (see $[5$, Theorem 11.14]).

Proof of Theorem 1.3: Note that Theorem 1.2 and (4) give

$$
\beta_{L^{2}}\left(m, \gamma_{2}, L^{p}\right) \approx\|m\|, \quad 1 \leqslant p \leqslant 2
$$

To obtain (ii) we simply use the following more general result.

THEOREM 3.5. If $Y$ has cotype $r<\infty$ and $Y^{*}$ has the RNP then

$$
\begin{aligned}
& \beta_{L^{2}(\nu)}\left(m, \gamma_{2}, Y\right) \approx\|m\|, \quad r=2 \\
& \beta_{L^{q}(\nu)}\left(m, \gamma_{q}, Y\right) \approx\|m\|, \quad q>r>2
\end{aligned}
$$

ProOF: We only prove (11). The other is exactly the same.

Suppose that $\left(A_{j}\right)$ is a sequence of pairwise disjoint sets. Since $m\left(A_{j}\right)$ is unconditionally convergent in $Y$, Lemma 3.4 implies that there exist $\left(\alpha_{j}\right) \in \ell^{q}$ and a sequence in $\left(y_{j}\right) \subset Y$ with $m\left(A_{j}\right)=\alpha_{j} y_{j}$ and

$$
\begin{array}{r}
\left(\sum_{j}\left|\alpha_{j}\right|^{q}\right)^{1 / q} \leqslant\left(\sup _{\left\|y^{*}\right\|=1} \sum_{j}\left|\left\langle m\left(A_{j}\right), y^{*}\right\rangle\right|\right)^{1 / q} . \\
\left(\sup _{\left\|y^{*}\right\|=1} \sum_{j}\left|\left\langle y_{j}, y^{*}\right\rangle\right|^{q^{\prime}}\right)^{1 / q^{\prime}}
\end{array}
$$

On the other hand if $u \in\left(L^{q}(\nu) \widehat{\otimes} Y\right)^{*}$, using (i) in Lemma 3.3, one has $u \in \Pi_{q^{\prime}}\left(Y, L^{q^{\prime}}\right)$. Therefore

$$
\begin{aligned}
\sum_{j}\left\|u\left(m\left(A_{j}\right)\right)\right\| & =\sum_{j} \mid \alpha_{j}\left\|u\left(y_{j}\right)\right\| \\
& \leqslant\left(\sum_{j}\left|\alpha_{j}\right|^{q}\right)^{1 / q}\left(\sum_{j}\left\|u\left(y_{j}\right)\right\|^{q^{\prime}}\right)^{1 / q^{\prime}} \\
& \leqslant \pi_{q^{\prime}}(u)\left(\sum_{j}\left|\alpha_{j}\right|^{q}\right)^{1 / q}\left(\sup _{\left\|y^{*}\right\|=1} \sum_{j}\left|\left\langle y_{j}, y^{*}\right\rangle\right|^{q^{\prime}}\right)^{1 / q^{\prime}} \\
& \leqslant C\|u\|_{\left(L^{q}(\nu) \hat{\otimes} Y\right) *}\|m\| .
\end{aligned}
$$

\section{Measures of Infinite $X$-SEMivariation}

We shall present now some necessary conditions to have bounded $X$-semivariation.

Proposition 4.1 . (i) Assume that $X \widehat{\bigotimes}_{\tau} Y$ is of finite cotype $q$. If $m: \Sigma$ $\rightarrow Y$ be a vector measure then

$$
\|m\|_{q} \leqslant C_{q} \beta_{X}(m, \tau, Y)
$$


for some constant $C_{q}$ independent of $m$.

In particular, if $X$ has finite cotype $q$ and $1 \leqslant p<\infty$ then

$$
\|m\|_{\max \{q, 2, p\}} \leqslant C \beta_{X}\left(m, \Delta_{p}, L^{p}(\mu)\right) .
$$

(ii) Let $1 \leqslant q<\infty$, let $\nu$ be a finite measure for which there exists a sequence of pairwise disjoint sets with $\nu\left(B_{j}\right)>0$ and let $m: \Sigma \rightarrow Y$ be a vector measure. Then

$$
\|m\|_{q} \leqslant C_{q} \beta_{L^{q}(\nu)}\left(m, \gamma_{q}, Y\right)
$$

Proof: (i) Suppose that $\left(x_{j}\right)$ is a sequence in the unit ball of $X$ and a sequence of pairwise disjoint sets $A_{j}$. Hence, for $0 \leqslant t \leqslant 1$, one has

$$
\left\|\sum_{j=1}^{k} r_{j}(t) x_{k} \otimes m\left(A_{j}\right)\right\|_{X \bar{\otimes}_{\tau} Y} \leqslant \beta_{X}(m, \tau, Y)
$$

where $r_{j}$ stands for the Rademacher sequence. Now integrate over $[0,1]$ and use the cotype estimate to get

$$
\left(\sum_{j=1}^{k}\left\|x_{k}\right\|^{q}\left\|m\left(A_{j}\right)\right\|^{q}\right)^{1 / q} \leqslant C_{q} \beta_{X}(m, \tau, Y) .
$$

Taking the sup over $\left(x_{j}\right)$ and $\left(A_{j}\right)$ one obtains the desired result.

Note that $L^{p}(\mu, X)$ has cotype equals $\max \{p, q, 2\}$.

(ii) Take $x_{j}=\left(\chi_{B_{j}}\right) /\left(\nu\left(B_{j}\right)^{1 / q}\right), \phi=\sum_{j=1}^{k} x_{j} \chi_{A_{j}}$ for some sequence of pairwise disjoint sets in $\Sigma$ and notice that, for any $A \in \Sigma$,

$$
\|\phi \otimes m(A)\|_{L^{q}(\nu, Y)}=\left(\sum_{j=1}^{k}\left\|m\left(A \cap A_{j}\right)\right\|^{q}\right)^{1 / q} .
$$

This gives the result.

COROLlary 4.2. Let $Y$ be infinite dimensional Banach space, $1 \leqslant q<2$ and $\nu$ be a finite measure for which there exists a sequence of pairwise disjoint sets with $\nu\left(E_{n}\right)>0$.

(i) There exist $Y$-valued measure such that $\beta_{L^{q}(\nu)}\left(m, \gamma_{q}, Y\right)=\infty$.

(ii) If $L^{p}(\mu)$ is infinite dimensional then there exist $L^{p}(\mu)$-valued measures $m$ such that $\beta_{L^{q}(\nu)}\left(m, \Delta_{p}, L^{p}(\mu)\right)=\infty$ for $1 \leqslant q<2$ and $q \geqslant p$.

Proof: (i) Select an unconditionally convergent series $\left(y_{n}\right)$ with $\sum_{k}\left\|y_{k}\right\|^{q}=\infty$ (this can be done for $1 \leqslant q<2$, see, for instance [5]).

Now we define the measure over $\mathbf{N}$ given by $m(\{k\})=y_{k}$. Clearly $\|m\|_{q}=\infty$ and therefore $\beta_{L^{q}(\nu)}\left(m, \gamma_{q}, Y\right)=\infty$ from (ii) in Proposition 4.1. 
(ii) follows from (i) and the estimate (3).

A very important example to analyse is $m_{p}: \Sigma \rightarrow L^{p}(\mu)$ given by $m_{p}(A)=\chi_{A}$. We shall see that these measures are enough to produce examples with $\beta_{L^{q}(\nu)}\left(m, \gamma_{q}, L^{p}(\mu)\right)$ $=\infty$ for $q<p$.

THEOREM 4.3. Let $\mu\left(\Omega_{1}\right)<\infty, \nu\left(\Omega_{2}\right)<\infty, X=L^{q}(\nu)$ and $Y=L^{p}(\mu)$. Then the $L^{p}(\mu)$-valued measure $m_{p}(A)=\chi_{A}$ has finite $L^{q}(\nu)$-semivariation in $L^{q}(\nu) \widehat{\otimes}_{\gamma_{q}} L^{p}(\mu)$ if and only if $L^{q^{\prime}}\left(\nu, L^{p^{\prime}}(\mu)\right) \subseteq L^{1}\left(\mu, L^{q^{\prime}}(\nu)\right)$.

Proof: Let $g: \Omega_{1} \times \Omega_{2} \rightarrow \mathbb{R}$ be such that

$$
\|g\|_{L q^{\prime}\left(\nu, L p^{\prime}(\mu)\right)}=\left(\int_{\Omega_{2}}\left(\int_{\Omega_{1}}|g(y, x)|^{p^{\prime}} d \mu(x)\right)^{q^{\prime} / p^{\prime}} d \nu(y)\right)^{1 / q^{\prime}}<\infty
$$

Note that the operator $v_{g}: L^{p}(\mu) \rightarrow L^{q^{\prime}}(\nu)$ becomes

$$
v_{g}(\psi)(y)=\int_{\Omega_{1}} g(y, x) \psi(x) d \mu(x),
$$

hence, we have $v_{g} \circ m_{p}(A)=\int_{A} g(y, x) d \mu(x)$ for all $A \in \Sigma_{1}$. This shows that $v_{g} \circ m_{p}$ is the $L^{q^{\prime}}(\nu)$-valued measure with Radon-Nikodym derivative $g(y,$.$) . Therefore$

$$
\left\|v_{g} \circ m_{p}\right\|_{1}=\int_{\Omega_{1}}\left(\int_{\Omega_{2}}|g(y, x)|^{q^{\prime}} d \nu(y)\right)^{1 / q^{\prime}} d \mu(x) .
$$

Now Theorem 2.2 shows that $m_{p}$ is of bounded $L^{q}(\nu)$-semivariation in $L^{q}(\nu) \widehat{\bigotimes}_{\gamma_{q}} L^{p}(\mu)$ if and only if there exists $C>0$ such that

$$
\int_{\Omega_{1}}\left(\int_{\Omega_{2}}|g(y, x)|^{q^{\prime}} d \nu(y)\right)^{1 / q^{\prime}} d \mu(y) \leqslant C\left(\int_{\Omega_{2}}\left(\int_{\Omega_{1}}|g(y, x)|^{p^{\prime}} d \mu(x)\right)^{q^{\prime} / p^{\prime}} d \nu(y)\right)^{1 / q^{\prime}} .
$$

That is to say $L^{q^{\prime}}\left(\nu, L^{p^{\prime}}(\mu)\right) \subset L^{1}\left(\mu, L^{q^{\prime}}(\nu)\right)$.

CoRollary 4.4. Let $1 \leqslant p<\infty$ and $m_{p}: \Sigma \rightarrow L^{p}(\mu)$ given by $m_{p}(A)=\chi_{A}$. Then $\beta_{L^{q}(\nu)}\left(m_{p}, \gamma_{q}, L^{p}(\mu)\right)<\infty$ for $p \leqslant q$.

Proof: Note that for $p \leqslant q$ one obviously has

$$
L^{q^{\prime}}\left(\nu, L^{p^{\prime}}(\mu)\right) \subset L^{q^{\prime}}\left(\nu, L^{q^{\prime}}(\mu)\right)=L^{q^{\prime}}\left(\mu, L^{q^{\prime}}(\nu)\right) \subset L^{1}\left(\mu, L^{q^{\prime}}(\nu)\right) .
$$

Apply now Theorem 4.3 .

Actually the previous result is also a consequence of the following general fact.

Proposition 4.5. Let $1 \leqslant p<\infty, X$ a Banach space and let $m: \Sigma \rightarrow L^{p}(\mu)$ be a positive vector measure, that is $m(A) \geqslant 0$ for all $A \in \Sigma$. Then

$$
\beta_{X}\left(m, \Delta_{p}, L^{p}(\mu)\right)=\|m\| .
$$

In particular, if $m$ is positive and $p \leqslant q$ then

$$
\beta_{L^{q}(\nu)}\left(m, \gamma_{q}, L^{p}(\mu)\right)=\|m\| .
$$


Proof: It is well-known that $\left(L^{p}(\mu, X)\right)^{*}=\left(L^{p}(\mu) \widehat{\otimes} X\right)^{*}$ can be identified with the space of $X^{*}$-valued measures in $V^{p^{\prime}}\left(\mu, X^{*}\right)$ (see [4]). In particular, if $u \in\left(L^{p}(\mu) \hat{\otimes} X\right)^{*}$ $C L\left(L^{p}(\mu), X^{*}\right)$ (see for instance [3]) there exists $\phi \in L^{p^{\prime}}(\mu)$ such that $\|\phi\|_{p^{\prime}}$ $\leqslant\|u\|_{\left(L^{p}(\mu) \hat{\otimes} X\right)}$. and satisfies that

$$
\|u(\psi)\| \leqslant \int_{\Omega} \phi(t) \psi(t) d \mu(t)
$$

for any positive function $\psi \in L^{p}(\mu)$. Therefore, if $\|u\|_{\left(L^{p}(\mu) \hat{\otimes} X\right)^{*}}=1$ then

$$
\begin{aligned}
\sum_{j=1}^{k}\left\|u\left(m\left(A_{j}\right)\right)\right\| & \leqslant\|\phi\|_{p^{\prime}} \int_{\Omega} \sum_{j=1}^{k} \frac{|\phi(t)|}{\|\phi\|_{p^{\prime}}} m\left(A_{j}\right)(t) d \mu(t) \\
& \leqslant \sup \left\{\sum_{j=1}^{k}\left|\left\langle\phi^{\prime}, m\left(A_{j}\right)\right\rangle\right|:\left\|\phi^{\prime}\right\|_{L^{p^{\prime}}}=1\right\}
\end{aligned}
$$

Hence $\left\|u_{f} \circ m\right\|_{1} \leqslant\|m\|$. Apply now Theorem 2.2.

In the case $X=L^{q}(\nu)$ and $p \leqslant q(4)$ allows us to conclude the proof.

We shall now see that the range of values in Theorem 4.3 is sharp.

LEMMA 4.6. If $p>q$ then there exists $f:[0,1]^{2} \rightarrow \mathbb{R}^{+}$such that

$$
\int_{0}^{1}\left(\int_{0}^{1} f(x, y)^{q} d y\right)^{p / q} d x<\infty
$$

and

$$
\int_{0}^{1}\left(\int_{0}^{1} f(x, y)^{p} d x\right)^{1 / p} d y=\infty
$$

Proof: Denoting $\beta=p / q>1$ and $g(x, y)=f(x, y)^{q}$ it suffices to find $g:[0,1]^{2}$ $\rightarrow \mathbb{R}^{+}$such that

$$
\int_{0}^{1}\left(\int_{0}^{1} g(x, y) d y\right)^{\beta} d x<\infty
$$

and

$$
\int_{0}^{1}\left(\int_{0}^{1} g(x, y)^{\beta} d x\right)^{1 / p} d y=\infty .
$$

Recall that the Hardy operator $T(\phi)(x)=(1 / x) \int_{0}^{x} \phi(y) d y$ is bounded on $L^{\beta}([0,1])$ for $\beta>1$ and define

$$
g(x, y)=\frac{1}{x} \chi_{[0, x]}(y) \phi(y)
$$

for a function $\phi \in L^{\beta}([0,1])$ to be chosen later.

Clearly

$$
\int_{0}^{1}\left(\int_{0}^{1} g(x, y) d y\right)^{\beta} d x=\|T(\phi)\|_{\beta}^{\beta} \leqslant\|T\|^{\beta}\|(\phi)\|_{\beta}^{\beta}
$$


On the other hand

$$
\begin{aligned}
\int_{0}^{1}\left(\int_{0}^{1} g(x, y)^{\beta} d x\right)^{1 / p} d y & =\int_{0}^{1} \phi(y)^{\beta / p}\left(\int_{y}^{1} \frac{d x}{x^{\beta}}\right)^{1 / p} d y \\
& \geqslant C \int_{0}^{1} \phi(y)^{\beta / p} \frac{1}{y^{(\beta-1) / p}} d y \\
& =C\left(\int_{0}^{1}\left(\frac{\phi(y)}{y^{1 / \beta^{\prime}}}\right)\right)^{\beta / p} d y \\
& \geqslant C\left(\int_{0}^{1} \frac{\phi(y)}{y^{1 / \beta^{\prime}}} d y\right)^{\beta / p} .
\end{aligned}
$$

Now select $\phi(y)=1 /\left(y^{1 / \beta}\right) \log (1 / y)$ to have $\phi \in L^{\beta}([0,1])$ and

$$
\int_{0}^{1} \frac{\phi(y)}{y^{1 / \beta^{\prime}}} d y=\int_{0}^{1} \frac{d y}{y \log (1 / y)}=\infty
$$

COROLlary 4.7. For $q<p$ the $L^{p}([0,1])$-valued measure $m_{p}(A)=\chi_{A}$ has infinite $L^{q}([0,1])$-semivariation in $L^{q}([0,1]) \widehat{\bigotimes}_{\gamma_{q}} L^{p}([0,1])$.

\section{REFERENCES}

[1] J.L. Arregui and O. Blasco, '(p,q)-summing sequences', J. Math. Anal. Appl. 247 (2002), 812-827.

[2] R. Bartle, 'A general bilinear vector integral', Studia Math. 15 (1956), 337-351.

[3] O. Blasco and P. Gregori, 'Lorentz spaces of vector-valued measures', J. London Math. Soc. 67 (2003), 739-751.

[4] N. Dinculeanu, Vector measures, International Series of Monographs in Pure and Applied Mathematics 95 (Pergamon Press, Oxford, New York, Toronto, 1967).

[5] J. Diestel, H. Jarchow and A. Tonge, Absolutely summing operators (Cambridge Univ. Press, Cambridge, 1995).

[6] J. Diestel and J.J. Uhl, Vector measures, Math. Surveys 15 (Amer. Math. Soc., Providence, R.I., 1997).

[7] B. Jefferies and S. Okada, 'Bilinear integration in tensor products', Rocky Mountain J. Math. 28 (1998), 517-545.

[8] B. Jefferies and S. Okada, 'Semivariation in $L^{p}$-spaces', Comment. Math. Univ. Carolin. 44 (2005), 425-436.

[9] B. Jefferies, S. Okada and L. Rodrigues-Piazza, ' $L^{p}$-valued measures without finite $X$-semivariation for $2<p<\infty$, (preprint).

Department of Mathematics

Universitat de Valencia

Burjassot 46100 (Valencia)

Spain

e-mail: oscar.blasco@uv.es 